\title{
Cytomorphological Studies in Dugesia Species of Iran
}

\author{
Ghorbani Behzad $^{1}$, Sheidai Masoud ${ }^{2, *}$ and Khazab Mahmood ${ }^{3}$ \\ ${ }^{1}$ Biological Department, Tehran University, Tehran, Iran \\ ${ }^{2}$ Biological Department, Shahid Beheshti University, Tehran, Iran \\ ${ }^{3}$ Genetic Section, Razi Research Institute, Karaj, Iran
}

Accepted September 2, 1998

\begin{abstract}
Summary Dugesia species of Karaj river in Iran were investigated from cytologial, and morphoanatomical point of view. Two different populations were studied. Individuals from Gajereh region possessed $2 n=25$ and those of Gachsar possessed $2 n=26$. They varied in details of karyotypes, GBanding pattern and morpho-anatomical features. Cluster analysis separated the two populations of Dugesia in different clusters. Two new species of Dugesia with new chromosome number having complete asexual reproduction are reported.
\end{abstract}

The fresh water planarians belong to the phylum Platyhelminthes, an old monophyletic group which branched from the rest of metazoans at an early stage and can be considered as the sister group of the other bilateral triploblastic organisms (Riutort et al. 1992, Addoutte and Philippe 1993). They possess a great morphological plasticity and regeneration power and are remarkable for the occurrence of local polyploid populations and the occasional presence of mixoploidy and mosaics having both diploid and triploid tissues (Hoshino et al. 1991, Benazzi and Lentati 1976, Oki et al. 1981).

Although there have been extensive cytological studies on planarian species in many countries (Hoshino et al. 1991, Benazzi and Lentati 1976, Oki et al. 1981, Tamura et al. 1988, Ribas et al. 1988), there has been no report from Iran. The present article is the first report on cytotaxonomy of Dugesia species from Iran.

\section{Materials and methods}

Dugesia species were collected from Gajereh and Gachsar regions of The Karaj river during 1995-1997. Specimens were brought to lab (temperature 4-8 $8^{\circ}$ ) and kept in the glass vials containing fresh well aerated water.

For karyotyping blastoma of the specimens were used. For this purpose; specimens were first cut from the head and tail regions and allowed to regenerate. It would take about one month for complete regeneration; specimens (blastomas) were collected after 5-8 days of regeneration and treated with $1 \%$ colchicin for $3-4 \mathrm{hr}\left(4^{\circ} \mathrm{C}\right)$, then passed to hypotonic solution (double distilled water) for about $30-35 \mathrm{~min}\left(5-20^{\circ} \mathrm{C}\right)$. The specimens were kept in fixative (Carnoys fluid) for $30 \mathrm{~min}\left(4^{\circ} \mathrm{C}\right)$. After dropping air dryed slides were prepared by keeping them inside incubator $\left(37^{\circ} \mathrm{C}\right)$ for $30 \mathrm{~min}$. Staining was carried out by using $10 \%$ Gimsa solution in buffer phosphate $(\mathrm{v}: \mathrm{v})$ $(\mathrm{pH}=6.9)$ for $10 \mathrm{~min}$. Chromosomes were identified according to Levans terminology (Levan et al. 1964). Karyotypic analysis was performed on 20 well prepared metaphase plates in each population.

For G-Banding all the steps involved in simple staining were followed plus using tripsin and $70 \%$ ethanol.

\footnotetext{
* Corresponding author.
} 
Table 1. Characters and their coding

1. The body size: $\mathrm{mm}$

2. Auricles: Short $=1$, long $=2$

3. Color pattern of the ventral side: Monotonous bright (unpigmented) $=1$, faint transversal regular strips $=2$

4. Color pattern of the dorsal side: Perpharyngeal area dark (pigmented), caudal area bright $=1$, cephalic area bright, post-pharyngeal area dark, caudal area bright $=2$

5. Pattern of pharynx area from ventral side: Distinguished bright margin $=1$, distinguished dark margin $=2$,

6. Pattern of pharynx area from dorsal side: Distinguished with humdrum marginal zone $=1$, distinguished with striped margin $=2$

7. $2 \mathrm{n}: 25=1,26=2$

8. No. SAT-Chromosomes: $6,13=1,13,21=2$

9. Mean of temperature change in environment ${ }^{\circ} \mathrm{C}$

\section{Statistical analysis}

Karyotypic and morphological characters were coded (Table 1), standardized (mean $=0$, variance $=1)($ Chatfield and Collins 1995 , Sheidai et al. 1996a, b) and used for multivariate statistical analysis. Cluster analysis using WARD and Single linkage methods (Sheidai and Inamdar 1997, Sheidai et al. 1996c, 1998) were performed. Fit of the clusters to the original data was checked by computing cophenetic correlation (Rohlf 1987, Stanton et al. 1994) For multivariate analysis SPSS (Norusis 1988) and NTSYS (Rohlf 1987) softwares were used.

\section{Results and discussion}

Specimens collected from Gajereh region possessed two prominent large eyes on the head; close to each other. The elongated and not very thin auricles formed a completely triangular head. The body color in the ventral side was bright color (yellow, brown or green) with a uniform pattern. The pharynx was without pattern and stain, dorsal side was also bright in color with no pattern. The body size ranged from $5-10 \mathrm{~mm}$.

The mode of reproduction was asexual (fissiparity) throughout the year. They possessed $2 n=$ 25 chromosome number. All these characters indicate that Gajereh specimens belong to the genus Dugesia.

The specimens collected from Gachsar region possessed almost similar features as those of Gajereh with larger body size $(5-15 \mathrm{~mm})$, larger eyes and $2 \mathrm{n}=26$. The members of these two populations however varied in some of the morphological characters such as type of auricle, color pattern of the ventral side, etc. (Table 1; code 1=Gajereh, code $2=$ Gachsar).

\section{Karyotypic analysis}

Details of karyotypes are presented in Table 2 . The populations studied did not show any significant difference in their total chromatin length, but varied in type of chromosomes for example Gajereh possossed 3B type chromosomes while Gachsar possessed 4, etc. (Table 2). Both populations possessed two SAT-Chromosomes (Table 2). The secondary constrictions were present on the 

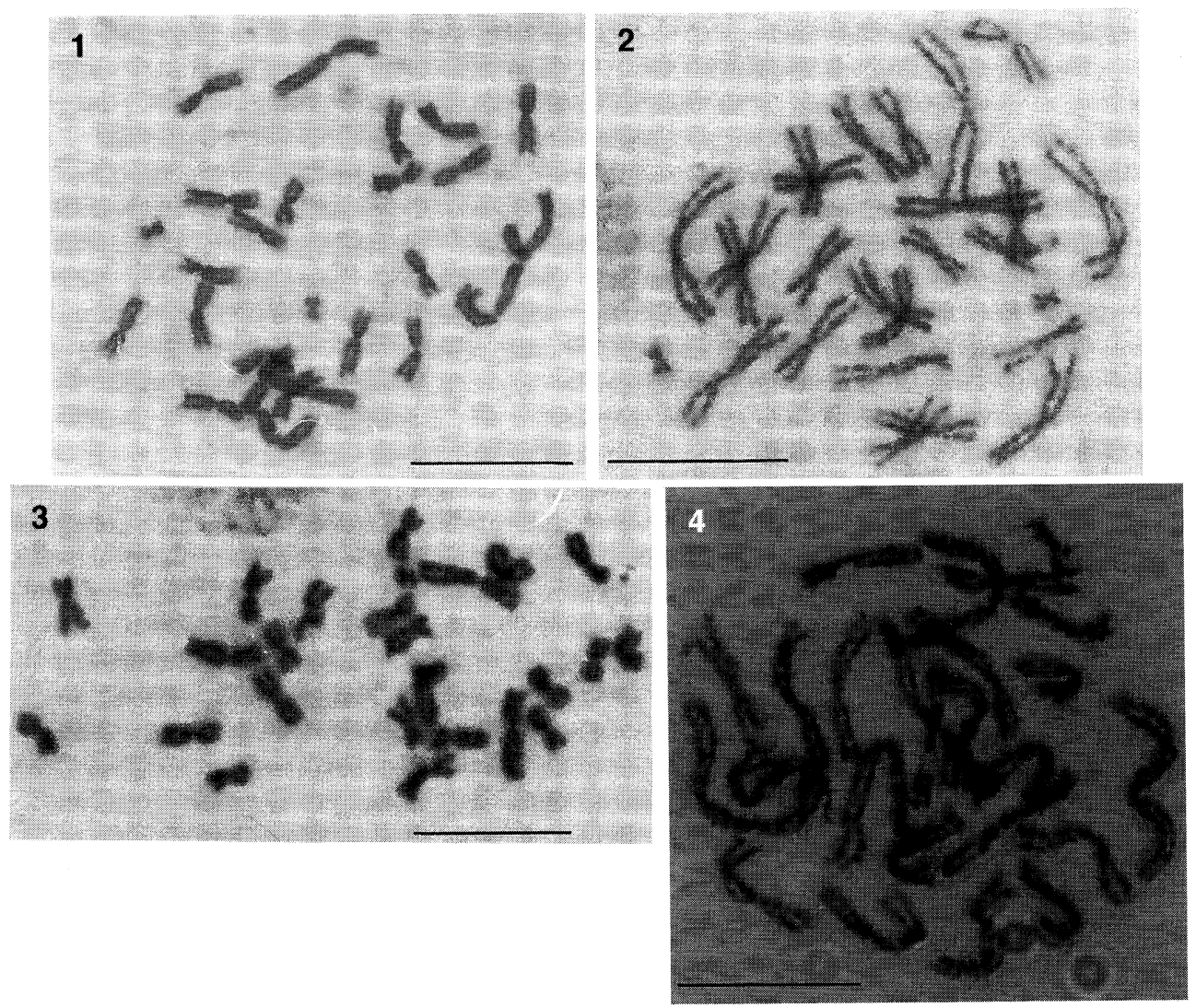

Fig. 1. Simple karyotype and G-Banding in Dugesia populations.

long arm of chromosome number 13 (in both populations) and on the short arm of chromosome number 6 (Gajereh) and 21 (Gachsar) (Figs. 1, 2).

G-Banding of the chromosomes also revealed differences in details of chromosomes in these two populations (Fig. 3). From karyotypic data it seems that numerical and structural changes of chromosomes have been occurred in genomic differentiation of these two populations.

The previous cytogenetical studies in $\mathrm{Du}$ gesia species have revealed the presence of $\mathrm{X}=4,7$ and 8 as the basic chromosome number (Benazzi and Lentati 1976, Oki et al. 1981), therefore $2 n=25$ and 26 are new reports.

Cluster analysis of cyto-morphological data indicated presence of two main clusters (Fig. 4). The first main cluster is comprised of specimens collected from Gajereh $(1,2)$ and the second main cluster is formed by specimens collected from Gachsar ( 3 to 6). A little variation
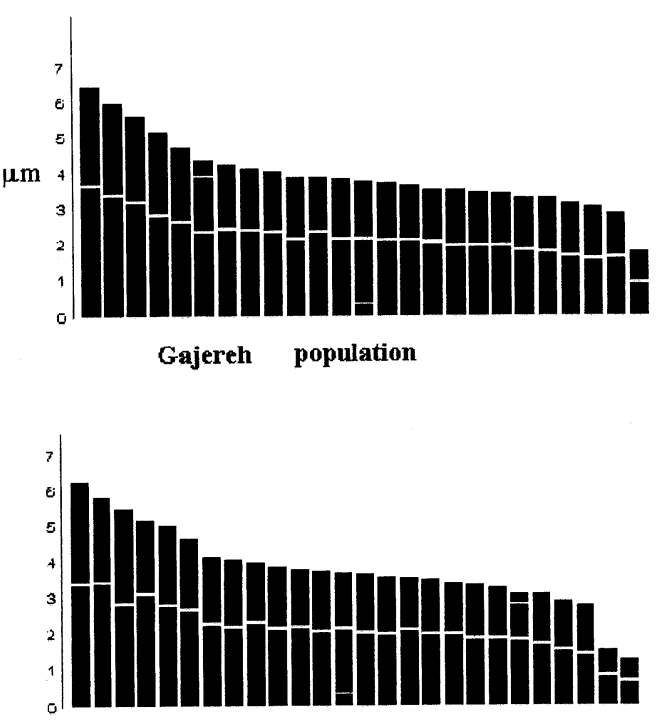

Gachsar population

Fig. 2. Ideograms of Dugesia Species. 

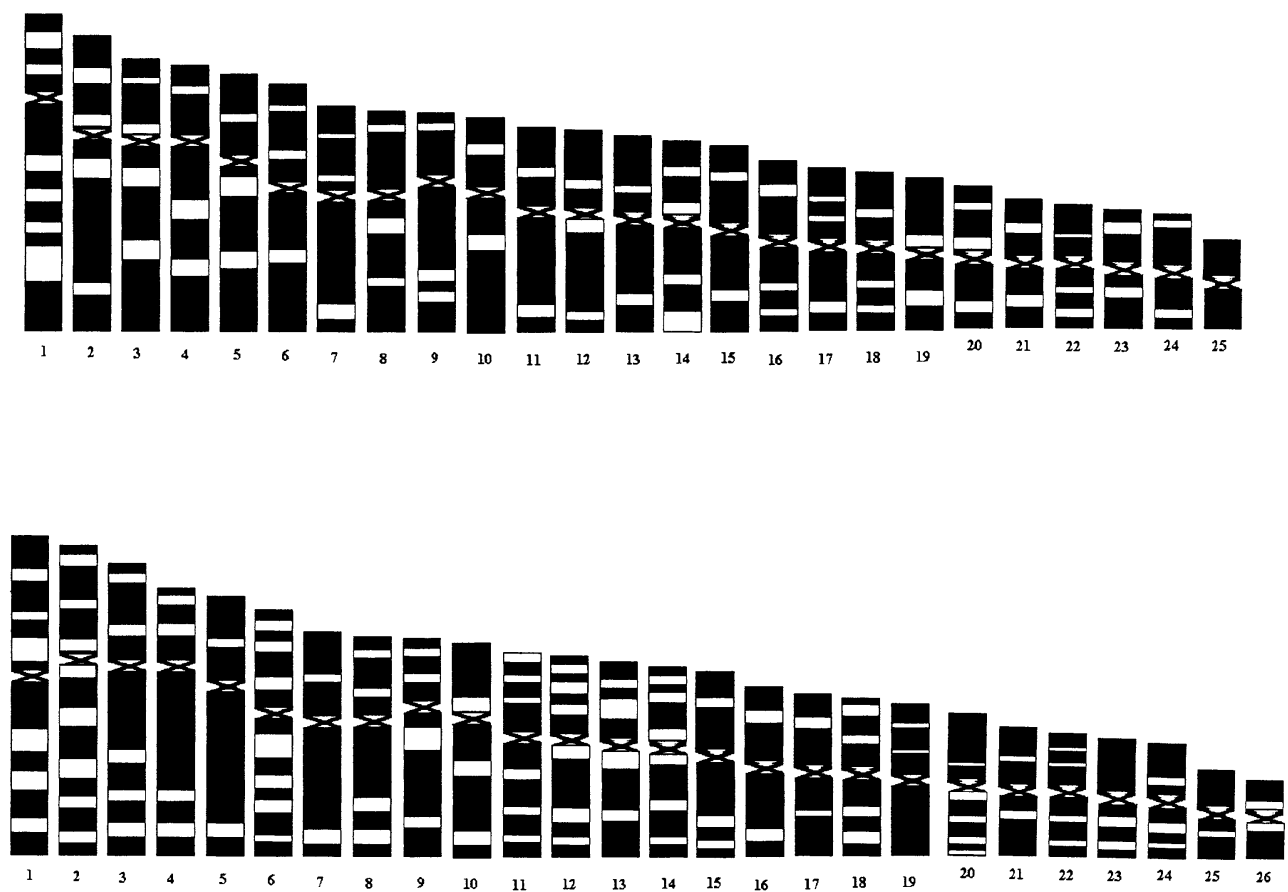

Fig. 3. G-Banding patterns in Dugesia populations; top: Gajereh, down Gachsar.

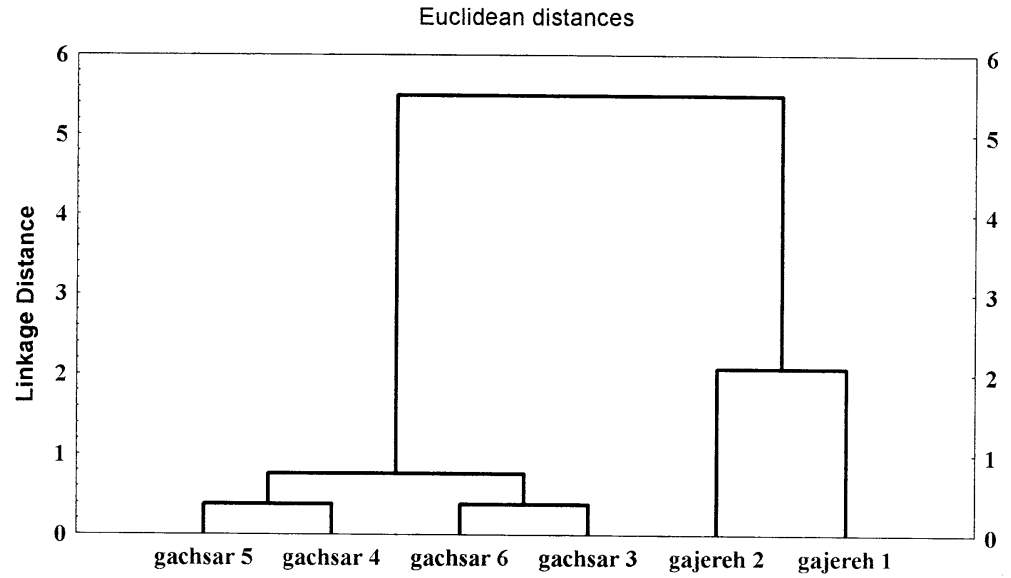

Fig. 4. Cluster analysis (Single linkage method) of cytomorphological data.

observed among members of each cluster is only due to body size differences. High cophenetic value $(r>0.80)$ obtained for cluster analysis supports distinctness of the clusters obtained (Rohlf 1987).

According to the present findings we suggest specimens collected from Gajereh and Gachsar to be treated as two new Dugesia species reported from Iran namely Dugesia Iranica and D. persica respectively.

\section{References}


from ribosomal RNA sequence analysis. In: Pichon, Y. (ed.) Comparative Molecular Neurobiology. Birhäuster Verlag. Basel: Switzerland: 1-34.

Benazzi, M. and Benazzi-Lentati, G. 1976. Platyhelminthes. In: B. John (ed.) Animal Cytogenetics. Vol. 1. Gebrüder Borntraeger. Berlin. Stuttgart: 1-182.

Chatfield, C. and Collins, A. J. 1995. Introduction to Multivariate Analysis. Chapman and Hall.

Hoshino, K., Ohnishi, K., Yoshida, W. and Shinozawa, T. 1991. Analysis of ploidy in a planarian by flow cytometry. Hydrobiologia 227: 175-178.

Levan, A. K., Fredga, K. and Sandberg, A. A. 1964. Nomenclature for centromeric position on chromosomes. Hereditas 52: 201-220.

Norusis, M. J. 1988. SPSS/PC. Advanced Statistics. SPSS Inc. Chicago.

Oki, I., Tamura, S., Yomayoshi, T. and Kawakatsu, M. 1981. Karyological and taxonomic studies of Dugesia japonica Ichikawa et Kawakatsu in the Far East. Hydrobiologia 84: 53-58.

Ribas, M., Pala, M., Vacca, R. A., Riutort, M. and Baguna, J. 1988. Taxonomic status of the western Mediterranean asexual populations of the Dugesia (D) gonocephala group. Morphological, karyological and biochemical data. Fortschr. Zool. 36: 129-138.

Riutort, M., Field, K. G., Turbeville, J. M., Raff, R. A. and Baguñà, J. 1992. Enzyme electrophoresis, ${ }^{18}$ S rRNA sequences, and levels of phylogenetic resolution among several species of freshwater planarians (Platyhelminthes, Tricladida, Paludicola). Can. J. Zool. 70: 1425-1439.

Rohlf, F. J. 1987. NTSYS-PC. Numerical Taxonomy and Multivariate Analysis System for the IBM-pc microcomputer (and compatible), ver. 1.4. Applied Biostatistics Inc.

Sheidai, M., Ahmadian, P. and Poorseyedy, Sh. 1996a. Cytological studies in Iran zira from three genus: Bunium, Carum and Cuminum. Cytologia 61: 19-25.

—, Maasoumii, A. R. and Pakravan, M. 1996b. Karyotypes of some Astaragalus taxa (Sec. Xiphidium Bge) from Iran. The nucleus. 38: 111-113.

—, Vojdani, P. and Alishah, O. 1996c. Karyological studies in Gossypium herbaceum cultivars of Iran. Cytologia 61: 365-374.

— and Inamdar, A. C. 1997. Cytomorphology of Asparagus taxa using multivariate statistical analysis. The nucleus. 40: $7-12$.

—, Vafai Tabar, M., Mirzai Nedoshan, H. and Hossininejad, Z. 1998. Cytogenetical studies in Gossypium hirsatum L. cultivars and their hybrids. Cytologia 63: 41-48.

Stanton, M., Stewart, J. McD., Percival, A. E. and Wendel, J. F. 1994. Morphological diversity and relationship in the Agenome cottons, Gossypium arbereum and G. herbaceum. Crop. Sc. 34: 519-527.

Tamura, S., Oki, I. and Kawakatsu, M. 1988. Karyological and taxonomic studies of Dugesia japonica from the Southwest Islands of Japan. Fortschr. Zool. 36: 123-128. 